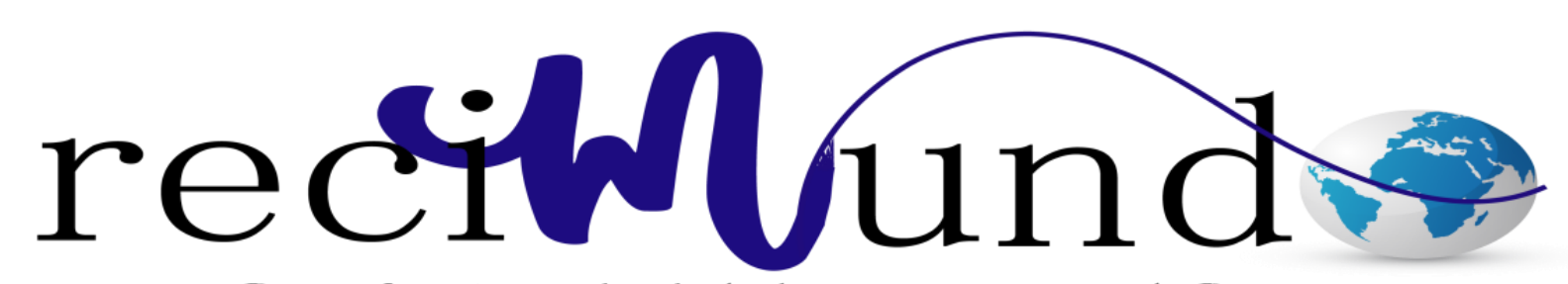

Revista Científica Mundo de la Investigación y el Conocimiento

Verónica Alexandra Muñoz Avilés a ; Cristina Dennisse Alencastro Sotovilla ${ }^{\text {b; }}$ María Belén Zhindón Astudillo ${ }^{\text {c; }}$ Diana Cristina Ordoñez Serpa ${ }^{\mathrm{d}}$

Tratamiento de disentería amebiana

Amoebic dysentery treatment

Revista Científica Mundo de la Investigación y el Conocimiento. Vol. 3 núm.3. Esp., noviembre, ISSN: 2588-073X, 2019, pp. 38-51

DOI: $10.26820 /$ recimundo/3.(3.Esp).noviembre.2019.38-51

URL: http://recimundo.com/index.php/es/article/view/602

Código UNESCO: 3205 Medicina Interna

Tipo de Investigación: Artículo de Revisión

(C) RECIMUNDO; Editorial Saberes del Conocimiento, 2019

Recibido: 15/09/2019

Aceptado: 23/10/2019

Publicado: 30/11/2019

Correspondencia: verito456@yahoo.com
a. Médico; Investigador Independiente; Guayaquil, Ecuador; verito456@yahoo.com
b. Médico; Investigador Independiente; Guayaquil, Ecuador; cdalencastro@gmail.com
c. Médico; Investigador Independiente; Guayaquil, Ecuador; belenzhindon11@ hotmail.com
d. Médico; Investigador Independiente; Guayaquil, Ecuador; dicrior@ hotmail.es 


\section{Tratamiento de disentería amebiana}

Vol. 3, núm. 3 Esp., (2019)

Verónica Alexandra Muñoz Avilés; Cristina Dennisse Alencastro Sotovilla; María Belén

Zhindón Astudillo; Diana Cristina Ordoñez Serpa

\section{RESUMEN}

La amebiasis es una infección intestinal causada por el protozoario (parásito) Entamoeba histolytica. Frecuentemente se presente como asintomática, no obstante, puede presentarse como disentería amebiana, e incluso evolucionar a un absceso hepático amebiano. En raras ocasiones, la amebiasis puede afectar áreas extra intestinales tales como los pulmones, el corazón o el cerebro. Representa la segunda causa de muerte por enfermedad parasitaria en todo el mundo. El objetivo fundamental de la presente investigación es plasmar el tratamiento de la Amebiasis. La Amebiasis más frecuente es la asintomática y su tratamiento se lleva a cabo con amebicidas intraluminales, entre los principales: la paramomicina y el iodoquinol. Para el caso de las amebiasis intestinales y hepáticas está indicado administrar amebicidas sistémicos como el metronidazol y posteriormente intraluminales. En casos infrecuentes y algunas complicaciones es requerida la intervención quirúrgica. De la revisión efectuada se concluye que, un diagnóstico preciso y un tratamiento farmacológico adecuado son la piedra angular para evitar complicaciones. La importancia de la unificación de criterios médicos compilados en guías de prácticas clínicas, es fundamental en el manejo de la enfermedad, lo cual aumenta las posibilidades de obtener mejores resultados que limiten la morbilidad y mortalidad.

Palabras Claves: Tratamiento; Amebiasis; Entamoeba; Histolytica; Disentería. 


\section{Tratamiento de disentería amebiana}

Vol. 3, núm. 3 Esp., (2019)

Verónica Alexandra Muñoz Avilés; Cristina Dennisse Alencastro Sotovilla; María Belén Zhindón Astudillo; Diana Cristina Ordoñez Serpa

\section{ABSTRACT}

Amebiasis is an intestinal infection caused by the protozoan (parasite) Entamoeba histolytica. Frequently it appears as asymptomatic, however, it can present as amoebic dysentery, and even evolve into an amoebic liver abscess. Rarely, amebiasis can affect extra intestinal areas such as the lungs, heart or brain. It represents the second cause of death from parasitic disease worldwide. The main objective of this research is to capture the treatment of Amebiasis. The most frequent Amebiasis is asymptomatic and its treatment is carried out with intraluminal amebicides, among the main ones: paramomycin and iodoquinol. In the case of intestinal and hepatic amebiasis it is indicated to administer systemic amebicides such as metronidazole and subsequently intraluminal. In infrequent cases and some complications surgical intervention is required. From the review carried out, it is concluded that an accurate diagnosis and adequate pharmacological treatment are the cornerstone to avoid complications. The importance of the unification of medical criteria compiled in clinical practice guidelines is fundamental in the management of the disease, which increases the chances of obtaining better results that limit morbidity and mortality.

Keywords: Treatment; Amebiasis; Entamoeba; Histolytica; Dysentery. 


\section{Tratamiento de disentería amebiana}

Vol. 3, núm. 3 Esp., (2019)

Verónica Alexandra Muñoz Avilés; Cristina Dennisse Alencastro Sotovilla; María Belén Zhindón Astudillo; Diana Cristina Ordoñez Serpa

\section{Introducción.}

La diarrea es unos principales problemas de salud en todo el mundo, especialmente en los países menos desarrollados. Sus causas pueden ser diversas entre las que destacan las infecciones intestinales por parásitos como las más frecuentes, y de este grupo las ocasionadas por las amebas.

La Secretaría de Salud de México (2014) explica al respecto, que la amebiasis es una infección intestinal causada por el protozoario (parásito) Entamoeba histolytica, el cual existe en dos etapas: como quiste que origina la forma infecciosa y una etapa de trofozoíto causante de la enfermedad invasiva. Aproximadamente 500 millones de personas se infectan anualmente en todo el mundo. La amebiasis en su mayoría se presente como asintomática, no obstante, puede presentarse como disentería amebiana, e incluso evolucionar a un absceso hepático amebiano. En raras ocasiones, la amebiasis puede afectar áreas extra intestinales tales como los pulmones, el corazón o el cerebro. Esta enfermedad "representa la segunda causa de muerte por enfermedad parasitaria en todo el mundo”. (p. 9).

Es importante resaltar que la disentería definida como una enfermedad independiente, es una de las causas más importantes de la infección amebiana, que consiste en la inflamación del intestino, especialmente del colon, lo cual ocasiona diarrea con sangre y en algunos casos también acompañada de mocos. Pero no sólo puede ser ocasionada por parásitos como la Entamoeba histolytica que origina la Amebiasis, también puede ser causada por bacterias como en el caso de la Shigella. La disentería puede causar la muerte si no se trata de manera adecuada. El tratamiento de la disentería consiste en restituir los líquidos perdidos, pero en el caso de la 


\section{Tratamiento de disentería amebiana}

Vol. 3, núm. 3 Esp., (2019)

Verónica Alexandra Muñoz Avilés; Cristina Dennisse Alencastro Sotovilla; María Belén Zhindón Astudillo; Diana Cristina Ordoñez Serpa

disentería amebiana el tratamiento está orientado al parásito que la ocasiona: Entamoeba histolytica.

Algunos autores utilizan el término "disentería amebiana" en su conjunto para definir la amebiasis en general, dado que en la mayoría de sus casos esta infección está acompañada de diarrea con sangre que es una de los principales síntomas de la disentería. A los efectos de la presente investigación se hará referencia a la "disentería amebiana” como amebiasis acompañada de diarrea sanguinolenta y a la "disentería" como la enfermedad derivada de la infección por amebas con su conjunto de síntomas, anteriormente mencionados.

En este orden de ideas, el agente etiológico de la Amebiasis: la ameba Entamoeba histolytica, puede vivir en el intestino grueso (colon) sin causarle daño. En algunos casos, invade la pared del colon y causa colitis, disentería aguda o diarrea prolongada (crónica)". Es una enfermedad presente en todo el mundo y más común en áreas tropicales, especialmente en áreas que presentan condiciones de hacinamiento y deficiente salubridad. (Enciclopedia Médica ADAN, 2019)

En un intento por superar enfermedades como la amebiasis, la Organización de las Naciones Unidas para la Alimentación y la Agricultura (2014) y la Organización Mundial de la Salud (OMS), se han centrado inicialmente en los 10 parásitos transmitidos por los alimentos con mayor impacto a nivel mundial, creando el informe "Clasificación multicriterio para la gestión de riesgos de los parásitos transmitidos por los alimentos”, dentro del ranking de estos 10 parásitos se encuentra la Entamoeba histolytica. 


\section{Tratamiento de disentería amebiana}

Vol. 3, núm. 3 Esp., (2019)

Verónica Alexandra Muñoz Avilés; Cristina Dennisse Alencastro Sotovilla; María Belén Zhindón Astudillo; Diana Cristina Ordoñez Serpa

La diarrea es una de los principales síntomas del amebiasis. En virtud de lo cual, es necesario mencionar que las enfermedades diarreicas:

Son la segunda mayor causa de muerte de niños menores de cinco años. Son enfermedades prevenibles y tratables. Las enfermedades diarreicas matan a 525000 niños menores de cinco años cada año. Una proporción significativa de las enfermedades diarreicas se puede prevenir mediante el acceso al agua potable y a servicios adecuados de saneamiento e higiene. En todo el mundo se producen unos 1700 millones de casos de enfermedades diarreicas infantiles cada año. La diarrea es una de las principales causas de malnutrición de niños menores de cinco años. (Organización Mundial de la Salud, 2017)

Es de vital importancia diferenciar la Entamoeba histolytica de especies morfológicamente similares, con la finalidad de indicar el tratamiento apropiado y evitar un tratamiento que no se requiera, por cuanto esta situación podría favorecer el desarrollo de resistencia antiparasitaria.

La presente investigación tiene como propósito fundamental revisar y plasmar lo relacionado con el tratamiento del amebiasis: amebiasis asintomática, disentería amebiana y amebiasis extraintestinal.

\section{Materiales y Métodos.}

El presente estudio se desarrolló por medio del uso de computadores personales con conexión a internet, para buscar material documental, a los fines de revisar, analizar y exponer 


\section{Tratamiento de disentería amebiana}

Vol. 3, núm. 3 Esp., (2019)

Verónica Alexandra Muñoz Avilés; Cristina Dennisse Alencastro Sotovilla; María Belén Zhindón Astudillo; Diana Cristina Ordoñez Serpa

acerca del tratamiento de la Amebiasis, lo que enmarca la investigación en un diseño de investigación documental o bibliográfica.

Se limitó a la búsqueda y revisión de material bibliográfico accesible mediante el uso de diversas bases de datos, entre ellas: MedlinePlus, PubMed, Biblioteca Virtual de la Salud (BVS), Dialnet y SciELO, páginas oficiales del área de la salud como la Organización Mundial de la Salud, Organización de las Naciones Unidas para la Alimentación y la Agricultura, Secretaría de la Salud de México, entre otras.

Se llevó a cabo una búsqueda aleatoria y consecutiva, donde se usando los siguientes descriptores "tratamiento de la amebiasis", "amebiasis"; "disentería amebiana", "amebiasis asintomática" y "absceso amebiano", el resultado fue de más de un centenar de miles de registros bibliográficos filtrados bajo los criterios de idioma español, relevancia, correlación temática y fecha de publicación en los últimos diez años, sin descartar por tipo de material bibliográfico.

Asimismo, se le dio lectura crítica al material, para ser posteriormente analizado y plasmado en el contenido del presente trabajo.

\section{Resultados.}

Chacín-Bonilla (2012) en su trabajo menciona que la Organización Mundial de la Salud y la Organización Panamericana de la Salud recomiendan someter a tratamiento a todos los casos de infección por E. histolytica, inclusive a los portadores de quistes. En aquellos casos en que sea imposible diferenciar esta amiba de otras morfológicamente iguales, la recomendación consiste en no tratar a los casos asintomáticos, a los fines de evitar tratamientos que no se necesiten. La selección del fármaco y su vía de administración deben basarse en la "localización de los 


\section{Tratamiento de disentería amebiana}

Vol. 3, núm. 3 Esp., (2019)

Verónica Alexandra Muñoz Avilés; Cristina Dennisse Alencastro Sotovilla; María Belén Zhindón Astudillo; Diana Cristina Ordoñez Serpa

trofozoítos en la luz o pared intestinal o en tejidos extraintestinales y en otros factores como la edad, severidad del cuadro clínico, presencia de otros parásitos intestinales o condiciones mórbidas, disponibilidad de los fármacos, eficacia clínica...”, entre otras. (p. 304, 305)

Entrando en materia de tratamiento, Del Barco, Álvarez, \& López (2009) refieren que la amebiasis más frecuente es la asintomática y que su tratamiento se lleva a cabo con amebicidas intraluminales, tales como: la paramomicina, furoato de diloxanida y el iodoquinol. Para el caso de las amebiasis intestinales y hepáticas está indicado administrar amebicidas sistémicos como el metronidazol y posteriormente intraluminales. Algunos casos pueden precisar de técnicas quirúrgicas (megacolon tóxico y abcesos hepáticos). (p. 19)

Por su parte, Alvarado et al. (2019) menciona que la colitis invasiva es tratada, por lo general, con metronidazol, tinidazol (TNZ), omidazol, nitaxozanida, entre los principales fármacos, ya sea como tratamiento único o en administración secuencial. Los autores concluyeron en su estudio que "en pacientes con Leucemia la administración de TNZ durante cinco días a la dosis de 2 g/día v.o. e iniciado al tiempo que la quimioterapia comienza, previene la aparición de amebiasis invasiva en estos pacientes”. (p. S33, S37).

El Instituto Nacional de Seguridad e Higiene en el Trabajo de España (2015) explica que:

La Entamoeba histolytica es un protozoo perteneciente al filo Sarcomastigophora, cuyo ciclo de vida comprende dos estadios: la forma invasiva vegetativa ameboide (trofozoíto) y la forma de resistencia e infectante (quiste). Antimicrobianos Frente a los trofozoítos: metronidazol (se han observado resistencias), derivados del nitroimidazol, 


\section{Tratamiento de disentería amebiana}

Vol. 3, núm. 3 Esp., (2019)

Verónica Alexandra Muñoz Avilés; Cristina Dennisse Alencastro Sotovilla; María Belén Zhindón Astudillo; Diana Cristina Ordoñez Serpa

tinidazol, secnidazol, ornidazol, nitazoxanida, cloraquina y emetina. Frente a los quistes: paromomicina, fuorato de diloxanida y diyodohidroxi- quinoleína (yodoquinol). (p. 1, 4).

Asimismo, Medina et al. (2011) hacen referencia a la importancia de la diferenciación entre los tipos de amebas, con la finalidad de indicar el tratamiento adecuado. Mencionan que para la diferenciación de E. histolytica, ameba patógena, de E. dispar, ameba no patógena, la cual no precisa tratamiento, es necesario una PCR-RT, prueba que solo puede realizarse en algunos en centros especializados. Con relación al tratamiento de la ameba patógena ver tabla 1. (p. 79, 80) 


\section{Tratamiento de disentería amebiana}

Vol. 3, núm. 3 Esp., (2019)

Verónica Alexandra Muñoz Avilés; Cristina Dennisse Alencastro Sotovilla; María Belén Zhindón Astudillo; Diana Cristina Ordoñez Serpa

Tabla 1: Tratamiento de la Amebiasis

\begin{tabular}{|c|c|c|c|c|c|}
\hline Patógeno & Fármaco & Dosis & Intervalo & Vía & Duración \\
\hline \multirow{2}{*}{$\begin{array}{l}\text { Entamoeba } \\
\text { histolytica } \\
\text { (portador } \\
\text { asintomático) }\end{array}$} & Paromomicina & $25-35 \mathrm{mg} / \mathrm{kg} / \mathrm{día}$ & $8 \mathrm{~h}$ & $\mathrm{VO}$ & 7-10 días \\
\hline & Iodoquinol & 30-40 mg/kg/día & $8 \mathrm{~h}$ & VO & 20 días \\
\hline \multirow{4}{*}{$\begin{array}{l}\text { Entamoeba } \\
\text { histolytica } \\
\text { (colitis } \\
\text { amebiana) }\end{array}$} & Metronidazol & 30-50 mg/kg/día & $8 \mathrm{~h}$ & VO & 10 días \\
\hline & (o tinidazol) & $50-60 \mathrm{mg} / \mathrm{kg} / \mathrm{día}$ & $24 \mathrm{~h}$ & VO & 3 días \\
\hline & Paromomicina & $25-35 \mathrm{mg} / \mathrm{kg} / \mathrm{día}$ & $8 \mathrm{~h}$ & VO & 10 días \\
\hline & (o Iodoquinol) & 30-40 mg/kg/día & $8 \mathrm{~h}$ & VO & 20 días \\
\hline \multirow{4}{*}{$\begin{array}{l}\text { Entamoeba } \\
\text { histolytica } \\
\text { (absceso } \\
\text { hepático) }\end{array}$} & Metronidazol & 30-50 mg/kg/día & $8 \mathrm{~h}$ & VO & 10 días \\
\hline & (o tinidazol) & $25-30 \mathrm{mg} / \mathrm{kg} / \mathrm{día}$ & $8 \mathrm{~h}$ & IV & 10 días \\
\hline & Paromomicina & $25-35 \mathrm{mg} / \mathrm{kg} / \mathrm{día}$ & $8 \mathrm{~h}$ & VO & 10 días \\
\hline & (o Iodoquinol) & 30-40 mg/kg/día & $8 \mathrm{~h}$ & VO & 20 días \\
\hline
\end{tabular}

Fuente: Medina et al. (2011)

Nota: Con relación al tinidazol, es un antiprotozoario oral aprobado por la FDA para tratamiento de giardiasis, amebiasis intestinal y absceso hepático amebiano en niños > 3 años (máx.: 2 g/día). Es necesaria su obtención por Medicamentos Extranjeros. Siempre se tienen que tratar con dos fármacos: paromomicina o iodoquinol se administran posteriormente a metronidazol o tinidazol. Contraindicado el uso de corticoides. 


\section{Tratamiento de disentería amebiana}

Vol. 3, núm. 3 Esp., (2019)

Verónica Alexandra Muñoz Avilés; Cristina Dennisse Alencastro Sotovilla; María Belén Zhindón Astudillo; Diana Cristina Ordoñez Serpa

La Secretaría de Salud de México (2014) en su Guía de Práctica Clínica para el

Diagnóstico y tratamiento del Absceso Hepático Amebiano no complicado recomienda:

El fármaco más utilizado para tratar el absceso hepático amebiano es el metronidazol 500 a $750 \mathrm{mg}$ por vía oral o intravenosa, tres veces al día durante 7 a 10 días, la misma dosis y duración, usada para la amebiasis intestinal. La tasa de curación es mayor de 90\%. La vía endovenosa no ofrece ninguna ventaja significativa, siempre y cuando el paciente pueda tomar medicamentos por vía oral y no tenga deficiencias en la absorción del intestino delgado. El metronidazol deberá usarse como fármaco de primera línea a una dosis de 30 a $50 \mathrm{mg} / \mathrm{Kg} /$ día si es $\mathrm{VO}$ y de $7.5 \mathrm{mg} / \mathrm{Kg} / \mathrm{dosis}$ si es endovenosa. Si es posible se utilizará VO. El tiempo de administración deberá ser entre 7 y 10 días. Fármacos como tinidazol u ornidazol pueden ser utilizados como segunda línea en caso de intolerancia al metronidazol. En presencia de intolerancia a la ingesta de metronidazol, se deberá utilizar tinidazol u ornidazol a una dosis de 60 mg por kilo/ día, máximo 2 grs. por diez días. Se puede utilizar nitazoxanida cuando existe intolerancia a los imidazoles. En pacientes que responden lentamente al metronidazol o tienen recaídas, la punción percutánea y/o un tratamiento prolongado de metronidazol debe ser considerado. Por lo que el drenaje radiológicamente guiado ha probado ser simple y efectivo; actualmente es considerado el estándar de oro con rangos de éxito entre 80 y 87 \%. Dejando la laparotomía solo para casos en los que se sospeche ruptura del absceso a la cavidad peritoneal o no se cuente con los recursos para punción percutánea o cirugía laparoscópica. (p. 20 - 23). 


\section{Tratamiento de disentería amebiana}

Vol. 3, núm. 3 Esp., (2019)

Verónica Alexandra Muñoz Avilés; Cristina Dennisse Alencastro Sotovilla; María Belén Zhindón Astudillo; Diana Cristina Ordoñez Serpa

Por último, Ramiro, Morán, \& Ximénez (2013) resaltan que no debe darse tratamiento antiamibiano sin antes comprobar que realmente existe amibiasis invasora intestinal o extraintestinal, y mucho menos, en los casos en que el individuo sea portador asintomático de dicha enfermedad. En el caso de amibiasis intestinal carente de disentería, es necesario indicar un antiamibiano no absorbible (diyodohidroxiquinoleina o la paromicina), igualmente puede indicarse uno absorbible (nitazoxamida). En aquellos casos de amibiasis intestinal con disentería, el tratamiento se debe iniciar con metronidazol por vía oral, $500 \mathrm{mg}$ tres veces al día durante 10 días. Cuando se requiera puede administrarse por vía endovenosa, con una dosis de $750 \mathrm{mg}$ dos veces al día. En los casos de absceso hepático, debe indicarse metronidazol por vía parenteral dos veces al día, $750 \mathrm{mg}$. Es de gran importancia mantener la vigilancia ante la posibilidad de ruptura del absceso, en cuyo caso "debe plantearse al paciente la necesidad de realizar drenaje del material purulento. La seguridad de este método por vía percutánea ha mejorado mucho al auxiliarse del ultrasonido". (p. 32, 33).

\section{Conclusiones.}

De la revisión efectuada se concluye que, un diagnóstico preciso y un tratamiento farmacológico adecuado son la piedra angular para evitar complicaciones de la Amebiasis. La importancia de la unificación de criterios médicos compilados en guías de prácticas clínicas, es fundamental en el manejo de la enfermedad, lo cual aumenta las posibilidades de obtener mejores resultados que limiten la morbilidad y mortalidad.

El tratamiento de la Amebiasis asintomática se lleva a cabo con amebicidas intraluminales, entre los principales Iodoquinol y Paramomicina. Mientras que el tratamiento de 


\section{Tratamiento de disentería amebiana}

Vol. 3, núm. 3 Esp., (2019)

Verónica Alexandra Muñoz Avilés; Cristina Dennisse Alencastro Sotovilla; María Belén Zhindón Astudillo; Diana Cristina Ordoñez Serpa

primera línea para la amebiasis intestinal y hepáticas es el metronidazol (amebicida sistémico) y posteriormente la administración de intraluminales. En un mínimo porcentaje de casos se requiere de procedimientos quirúrgicos.

Es fundamental la búsqueda novedosos fármacos amebicidas debido a la toxicidad del metronidazol y la resistencia de algunos protozoos intestinales a este fármaco, por lo que resulta imperioso realizar nuevos estudios clínicos a los fines de validar el uso de otros fármacos en el tratamiento de la amibiasis.

\section{Bibliografía.}

Alvarado, M., Arana, L., Álvarez, J., De la Peña, J., Mena, V., Ortiz, M., . . Pérez, J. (19 de Marzo de 2019). Eficacia del tinidazol en la profilaxis terapéutica de la colitis amebiana en pacientes con leucemia aguda de novo que reciben quimioterapia intensiva. Gaceta Médica de México, 155(1), S32-S37.

Chacín-Bonilla, L. (2012). Farmacoterapia actual de la amibiasis, avances en nuevas drogas y diseño de una vacuna. Investigación Clínica, 53(3), 301-314.

Del Barco, Ó., Álvarez, P., \& López, R. (2009). Parasitosis Intestinal. Guía de Práctica Clínica, Servicio Andaluz de Salud, Consejería de Salud y Familias, Andalucía.

Enciclopedia Médica ADAN. (02 de Octubre de 2019). Amebiasis. Obtenido de Medline Plus: https://medlineplus.gov/spanish/ency/article/000298.htm

Instituto Nacional de Seguridad e Higiene en el Trabajo de España. (2015). Entamoeba histolytica. Ministerio de Trabajo, Migraciones y Seguridad Laboral de España. Madrid: DataBio.

Medina, A., Mellado, M., García, M., Piñeiro, R., \& Martín, P. (2011). Parasitosis intestinales. En S. E. Pediátrica, Protocolos diagnóstico-terapéuticos de la Asociación Española de Pediatría: Infectología pediátrica (Vol. 3, págs. 77-88). Madrid, España: ERGON.

Organización de las Naciones Unidas para la Alimentación y la Agricultura. (01 de Julio de 2014). Identifican los diez principales parásitos transmitidos por los alimentos. Recuperado el 26 de Octubre de 2019, de Organización de las Naciones Unidas para la Alimentación y la Agricultura: http://www.fao.org/news/story/es/item/237578/icode/ 


\section{Tratamiento de disentería amebiana}

Vol. 3, núm. 3 Esp., (2019)

Verónica Alexandra Muñoz Avilés; Cristina Dennisse Alencastro Sotovilla; María Belén Zhindón Astudillo; Diana Cristina Ordoñez Serpa

Organización Mundial de la Salud. (02 de Mayo de 2017). Enfermedades diarreicas. Obtenido de Organización Mundial de la Salud: https://www.who.int/es/news-room/factsheets/detail/diarrhoeal-disease

Ramiro H., M., Morán, P., \& Ximénez G., C. (2013). Amebiasis, la enfermedad, su diagnóstico y tratamiento. Revista Ciencia, 62(2), 26-33.

Secretaría de Salud de México. (2014). Diagnóstico y tratamiento del Absceso Hepático Amebiano no. Secretaría de Salud de México, Coordinación de Unidades Médicas de Alta Especialidad. Cuauhtémoc: División de Excelencia Clínica.

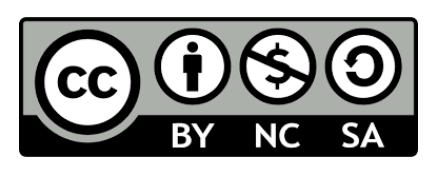

\section{RECONOCIMIENTO-NOCOMERCIAL-COMPARTIRIGUAL}

CC BY-NC-SA

ESTA LICENCIA PERMITE A OTROS ENTREMEZCLAR, AJUSTAR Y CONSTRUIR A PARTIR DE SU OBRA CON FINES NO COMERCIALES, SIEMPRE Y CUANDO LE RECONOZCAN LA AUTORÍA Y SUS NUEVAS CREACIONES ESTÉN BAJO UNA LICENCIA CON LOS MISMOS TÉRMINOS. 